\title{
Cortical Reorganization in Patients Recovered from Bell's Palsy: An Orofacial and Finger Movements Task-State fMRI Study
}

\author{
Jaeyoun Lee, ${ }^{1}$ Jun Yang, ${ }^{2}$ Chuanfu Li, ${ }^{3}$ Aihong Yuan, ${ }^{2}$ Hongli Wu, ${ }^{3}$ Anqin Wang, \\ Qiuju Xue, ${ }^{3}$ Tao Wang, ${ }^{3}$ Linying Wang, ${ }^{3}$ and Ting $\mathbf{G a o}^{3}$ \\ ${ }^{1}$ Department of Acupuncture, Anhui University of Chinese Medicine, Hefei, Anhui 230031, China
${ }^{2}$ Department of Acupuncture, The First Affiliated Hospital of Anhui University of Chinese Medicine, Hefei, Anhui 230031, China
${ }^{3}$ Laboratory of Digital Medical Imaging, The First Affiliated Hospital of Anhui University of Chinese Medicine, Hefei, \\ Anhui 230031, China
}

Correspondence should be addressed to Jun Yang; yangjunacup@126.com

Received 25 October 2016; Accepted 7 December 2016

Academic Editor: Toshiyuki Fujiwara

Copyright (C) 2016 Jaeyoun Lee et al. This is an open access article distributed under the Creative Commons Attribution License, which permits unrestricted use, distribution, and reproduction in any medium, provided the original work is properly cited.

\begin{abstract}
Objective. To explore cortical reorganization of patients recovered from Bell's palsy (BP) by task-state functional magnetic resonance imaging (fMRI) during finger and orofacial movements and provide more evidence for acupuncture clinical treatment of BP. Methods. We collected 17 BP patients with complete clinical recovery (BP group) and 20 healthy volunteers (control group) accepted the task-state fMRI scans with lip pursing movements and finger movements, respectively. Results. It was found that there were significant differences of brain functional status between the two groups. Conclusions. The results showed that there was cortical reorganization in the brain of patients recovered from BP after acupuncture treatment, which also suggested the relationship between the hand motor areas and facial motor areas of BP patients.
\end{abstract}

\section{Introduction}

Cortical reorganization, also called "cortical plasticity," is the ability of the cortex to adapt to changing circumstances and new information. Multiple studies demonstrated that functional plasticity occurred in various diseases, including brain lesions [1-3] and peripheral nerve lesions [4]. Bell's palsy is an acute, idiopathic, and unilateral paralysis of the face with a pure peripheral deafferentation and dysfunction of the facial nerve $[5,6]$, which is a common condition affecting approximately 20-35/100 000 people [7]. It is usually treated by medicine, surgical operation, acupuncture, and other clinical methods [8-11]. Previous studies have provided evidence that cortical reorganization played an important role in the recovery of Bell's palsy. For example, Rijntjes et al. used positron emission tomography (PET) and transcranial magnetic stimulation (TMS) to detect cortical reorganization in patients with facial palsy, which demonstrated that facial motor deafferentation leads to an enlargement and extension of the cortical hand field into the face area [12]. TMS was also used in facial paralysis with the task of tongue skills. The result showed the facial motor region was invaded by the neighboured tongue motor area bilaterally.

Functional magnetic resonance imaging (fMRI) is a popular radiological technique to investigate pathological mechanism in disease progression [13]. It has been used to detect patients with facial palsy recovery. Wu et al. [10] showed changed functional connectivity in the acute stage and subsequent reorganization during the recovery of the $\mathrm{BP}$ with resting fMRI. Hu et al. [14] found that increasing functional connectivity of the anterior cingulate cortex during the course of recovery from Bell's palsy might be related to the cortical reorganization.

$\mathrm{BP}$ is a disease of peripheral deafferentation and dysfunction of the facial nerve. It is a leading disorder of facial motor function. How does the cortical reorganization in facial motor area of patients recovered from BP and what is its relationship to hand motor area? With this aim, we detected cortical reorganization in recovery BP patients with two different motor tasks, the finger and lip pursing movements. 
TABLE 1: Information of patients recovered from BP.

\begin{tabular}{|c|c|c|c|c|c|}
\hline Number & Sex & Age & Paretic side & HBS (before treatment) & Duration (days) \\
\hline mr76788 & Female & 39 & Left & 5 & 99 \\
\hline mr74727 & Female & 45 & Left & 5 & 38 \\
\hline mr79842 & Female & 42 & Left & 4 & 102 \\
\hline mr76789 & Male & 43 & Right & 3 & 38 \\
\hline mr79877 & Male & 48 & Left & 3 & 54 \\
\hline mr79577 & Female & 28 & Right & 4 & 35 \\
\hline $\operatorname{mr} 81658$ & Female & 28 & Left & 4 & 38 \\
\hline mr83703 & Male & 26 & Right & 4 & 77 \\
\hline $\operatorname{mr93120}$ & Male & 26 & Left & 4 & 143 \\
\hline $\operatorname{mr103930}$ & Female & 25 & Right & 4 & 273 \\
\hline mr93076 & Male & 49 & Left & 4 & 49 \\
\hline mr95181 & Male & 26 & Right & 3 & 43 \\
\hline mr102581 & Male & 30 & Left & 4 & 178 \\
\hline $\operatorname{mr102582}$ & Female & 24 & Right & 3 & 68 \\
\hline mr102423 & Female & 44 & Right & 3 & 44 \\
\hline mr114175 & Male & 46 & Left & 3 & 146 \\
\hline mr111924 & Female & 27 & Right & 3 & 67 \\
\hline
\end{tabular}

\section{Materials and Methods}

2.1. Subjects. All subjects recruited in this study were divided into two groups, recovered palsy group and healthy control group. The recovered palsy group was composed of 17 cases of patients recovered from BP (all right-handed, female 9, male 8 , as shown in Table 1), who were the out-and-in patients from the First Affiliated Hospital of Anhui University of TCM (traditional Chinese medicine). They had been assessed as clinical recovery after acupuncture treatment by the HouseBrackmann facial nerve grading system (HBS) (House and Brackmann, 1985) [15]. HBS has been mostly widely used in studies of peripheral facial nerve palsy in recent years. This scale ranges from 1 , representing normal facial movements, to 6 , representing no movements. The control group was composed of 20 healthy volunteers (female 7 , male 13) from Anhui University of TCM and the hospital staff who were right-handed. All subjects were with no history of mental or neurological disease, with no obvious abnormality disorder or drug use, and with no obvious abnormality in brain structure. And, also, this study was approved by the Institutional Review Board of the First Affiliated Hospital of Anhui University of TCM, and written informed consent was obtained from each participant prior to the experiment.

2.2. Data Acquisition. The experiment was performed in the MRI room of the Medical Imaging Center, the First Affiliated Hospital of Anhui University of TCM. The Siemens Symphony 1.5 T MRI whole body scanner (Siemens Medical Systems, Germany) and standard head coil were used. Before experiment, the participants were requested to change clothes, rest, and then enter the scanning room after the whole body had been relaxed. They were instructed to lie down with eyes closed and to stay awake. All lights in the scanning room were turned off to avoid unwanted visual stimulation. We also should have expounded two task-state actions to the participants. During the entire scanning process, the subjects were asked to avoid psychological activity as far as possible.

Five sequences were scanned as follows: (1) pilot images; (2) T2-weighted images to rule out any disease of the brain; (3) EPI-BOLD; (4) T1-weighted 3D anatomical images: the sagittal position was taken, and total of 176 slices were scanned which covered the whole brain. The spoiled gradient echo sequence was used, with TR/TE/FA $=2100 \mathrm{~ms} / 3.93 \mathrm{~ms} / 13^{\circ}, \mathrm{FOV}$ of $250 \times 250 \mathrm{~mm}$, slice thickness $/$ spacing $=1.0 \mathrm{~mm} / 0.5 \mathrm{~mm}$, and resolution of $256 \times 256$. (5) Task-state fMRI took about 30 minutes to complete all of the data acquisition.

Task-State fMRI. Lip pursing movements and finger movements were selected as two task-state actions. EPI-BOLD was used with $\mathrm{TR} / \mathrm{TE} / \mathrm{FA}=3000 \mathrm{~ms} / 30 \mathrm{~ms} / 90^{\circ}$, slice thickness $/$ spacing $=3.0 \mathrm{~mm} / 0.75 \mathrm{~mm}$, FOV $192 \mathrm{~mm} \times 192 \mathrm{~mm}$, and FOV $64 \times 64 \mathrm{~mm}$. And the data of task-state scanning used block design, every block took 30 seconds, and all scanning of data of task-state fMRI took 6 minutes, 120 cardinal numbers of functional data. Every functional data acquisition took 3 seconds (TR), as shown in Figure 1.

2.3. Data Preprocessing. Data analysis was performed using the software of AFNI (http://afni.nimh.nih.gov/afni/) in the Laboratory of Digital Medical Imaging, the First Affiliated Hospital of Anhui University of TCM. Initially, the first 4 time points of the functional images were discarded to avoid the instability of the initial MRI signal and the remaining images were realigned to the first volume. Thereafter, the images were normalized to the standard Talairach atlas and then smoothed spatially using a $6 \mathrm{~mm}$ full width at half maximum (FWHM) Gaussian kernel to decrease spatial noise. The time 
TABLE 2: Intergroup analysis of areas when making finger movements.

\begin{tabular}{|c|c|c|c|c|c|c|}
\hline \multirow{2}{*}{ Region } & \multirow{2}{*}{ Side } & \multicolumn{3}{|c|}{ Talairach $(\mathrm{mm})$} & \multirow{2}{*}{$Z$} & \multirow{2}{*}{ Voxels } \\
\hline & & $x$ & $y$ & $z$ & & \\
\hline PCC & Right & -1.5 & 49.5 & 11.5 & 1.211 & 369 \\
\hline Precuneus & Left & 1.5 & 76.5 & 32.5 & 1.507 & 353 \\
\hline Transverse temporal gyrus & Left & 61.5 & 16.5 & 11.5 & 0.892 & 186 \\
\hline SI/MI & Right & -61.5 & 22.5 & 14.5 & 0.859 & 92 \\
\hline Middle temporal gyrus & Right & -64.5 & 16.5 & -6.5 & 0.661 & 83 \\
\hline CMA & Left & 1.5 & 19.5 & 41.5 & 0.562 & 80 \\
\hline Parahippocampal gyrus & Right & -19.5 & 1.5 & -9.5 & -0.733 & 71 \\
\hline
\end{tabular}

The threshold was set at $P=0.05, \alpha<0.05$, cluster $=61$ (corrected with Monte-Carlo method). PCC: posterior cingulate cortex; MI: primary motor cortex; SI: primary somatosensory cortex; CMA: cingulate motor area.

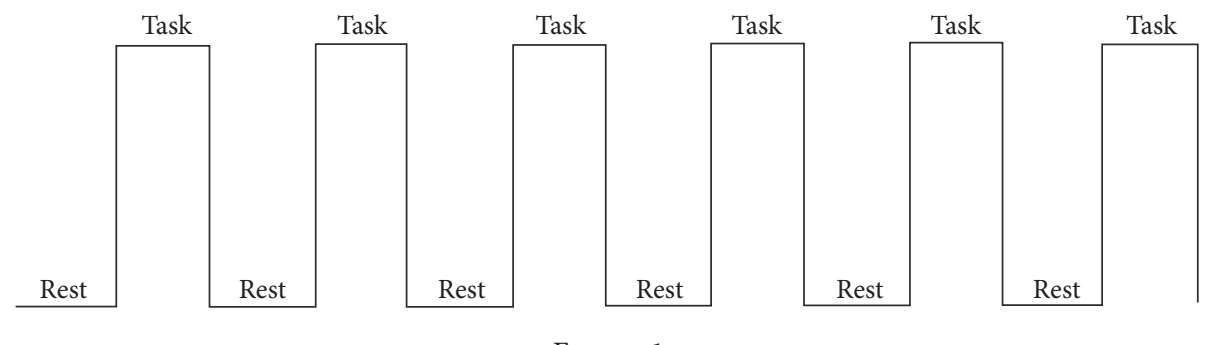

FIGURE 1

series from each voxel was detrended using the method of linear least squares to remove low-frequency noise and signal drift. For each subject, the preprocessed fMRI data were then submitted for analyses using the general linear model, and the coef value in the individual analysis results was extracted as the contrast image for further analysis. Before processing, individual data of right-sided facial palsy patients were flipped along $y$-axis so that all data could be processed unilaterally.

2.4. Intergroup Analysis. Before intergroup analysis, the individual data with head movements more than $2 \mathrm{~mm}$ or $2^{\circ}$ were excluded to avoid the possible influence of head movements on the results of the data analysis. As a result, 4 cases in the palsy recovered group were excluded. Intergroup comparison was performed with $3 \mathrm{dttest++}$ to investigate the variation of brain activation between patients and controls. The results of intergroup analysis were corrected using Monte-Carlo simulation, with $P=0.05, \alpha<0.05$, cluster $=61$. To detect the cortical functional reorganization in patients with facial paralysis, the regions of interest were extracted from the statistic activation maps from hand task experiment for controls and mouth task experiment for patients to get the intersection.

\section{Results}

3.1. General Information. 17 cases of BP group (mean age: 35.06 years, range: $20-70$ years) and 20 cases of healthy control group (mean age: 31.7 years, range: $20-70$ years) were not significantly different among subjects' age distributing and the sample size of two groups. To address the significant differences in cortical reorganization between different groups, the results of group analysis for each group were showed as follows. There were no subjects removed from data analysis. In the study, the data of right-sided BP patients were flipped along the y-axis so that all data could be processed unilaterally. So all patients can be considered the left-sided BP patients. The left activated areas were considered to be contralateral, and the right activated areas were ipsilateral.

3.2. Finger Movements Task-State. As shown in Table 2 and Figure 2, when performing finger movements compared with the healthy control group, it showed increased activation in the contralateral PCC (posterior cingulate cortex), MI (primary motor cortex), SI (primary somatosensory cortex), middle temporal gyrus and ipsilateral CMA (cingulate motor area), precuneus, and transverse temporal gyrus. There was decreased signal in the contralateral parahippocampal gyrus (Table 2, Figure 2).

3.3. Lip Pursing Movements Task-State. When making lip pursing movements compared with the healthy control group, it showed decreased activation in the ipsilateral culmen, CMA (posterior cingulate cortex), transverse temporal gyrus, SI (primary somatosensory cortex), precuneus, and contralateral superior occipital gyrus with lip pursing movements (Table 3, Figure 3).

\section{Discussion}

This is a report on the changes in the brain functional status of patients recovered from BP by movements taskstate functional MRI, which would provide more evidence for clinical treatment. With this objective, differences of 
TABLE 3: Intergroup analysis of areas when making lip pursing movements.

\begin{tabular}{|c|c|c|c|c|c|c|}
\hline \multirow{2}{*}{ Region } & \multirow{2}{*}{ Side } & \multicolumn{3}{|c|}{ Talairach $(\mathrm{mm})$} & \multirow{2}{*}{$Z$} & \multirow{2}{*}{ Voxels } \\
\hline & & $x$ & $y$ & $z$ & & \\
\hline Culmen & Left & 37.5 & 55.5 & -24.5 & -1.703 & 621 \\
\hline PCC & Left & 1.5 & 40.5 & 17.5 & -0.837 & 154 \\
\hline Transverse Temporal Gyrus/SI & Left & 61.5 & 13.5 & 11.5 & -1.342 & 121 \\
\hline Superior Occipital Gyrus & Right & -31.5 & 82.5 & 23.5 & -0.832 & 110 \\
\hline Precuneus & Left & 19.5 & 70.5 & 44.5 & -0.780 & 86 \\
\hline
\end{tabular}

The threshold was set at $P=0.05, \alpha<0.05$, cluster $=61$ (corrected with Monte-Carlo method). BA: Brodmann area; PCC: posterior cingulate cortex; SI: primary somatosensory cortex.

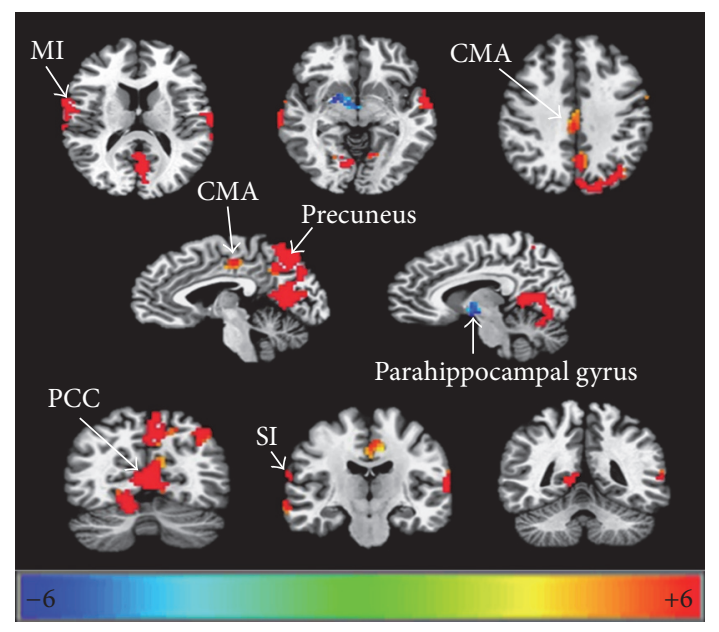

FIgURE 2: Cortical reorganization in the areas of palsy recovered group compared to healthy control group when conducting finger movements. $P=0.05, \alpha<0.05$, corrected with Monte-Carlo method. PCC: posterior cingulate cortex; MI, primary motor cortex; SI: primary somatosensory cortex; CMA: cingulate motor area.

brain functional status between the recovered palsy group and healthy control group were investigated in this study. Firstly, we would discuss two important questions, whether cortical reorganization existed or not and what cortical reorganization might imply.

4.1. Cortical Reorganization in Patients Recovered from BP. From results of this paper, it showed that there were significant differences of cortical function status between recovered palsy group and healthy control group during finger movements and lip pursing movements. Therefore, we could get the conclusion that cortical reorganization still existed in patients recovered from BP, or the brain functional status had not returned to the condition before the disease. Bell's palsy patients had been assessed as recovery when their grade of HBS was I; it just implied the clinical symptoms disappeared, but it did not mean cortical reorganization would return to status before the disease. Actually, previous studies have indicated that cortical reorganization in multiple related sensorimotor areas existed during the whole pathological stage of BP $[10,14,16]$. This study indicated cortical reorganization existed in the early recovery stage. In general,

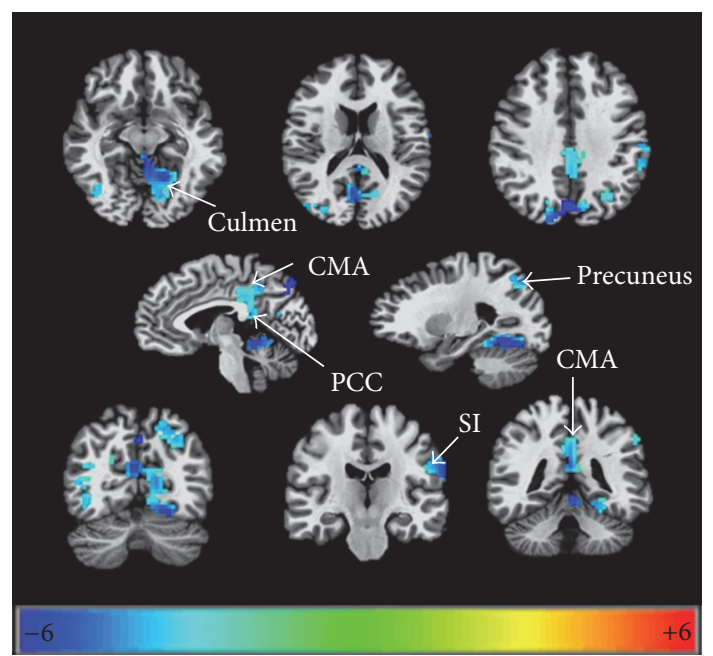

FIgURE 3: Cortical reorganization in the areas of palsy recovered group compared to healthy control group when conducting lip pursing movements. $P=0.05, \alpha<0.05$, corrected with Monte-Carlo method. PCC: posterior cingulate cortex; SI: primary somatosensory cortex.

patients will stop treatment after clinical symptoms disappear. This study also implied that perhaps patients should continue the treatment even after clinical recovery to enhance the thorough recovery of brain function.

\subsection{Differences between Recovered Palsy Group and Healthy} Control Group. In this study, significantly increased activation in posterior cingulate cortex (PCC), primary somatosensory cortex (SI), primary motor cortex (MI), and cingulate motor area (CMA) and decreased activation in parahippocampal gyrus during finger movements were found. And we also observed decreased signal in primary somatosensory cortex (SI), posterior cingulate cortex (PCC), precuneus and culmen during lip pursing movements. These activated areas, which were associated with hand and orofacial movements, were components of a network that controlled the cortical and subcortical representation of voluntary facial movements, which were reported in many studies $[15,17$, 18]. The functional network of the human brain was pretty complicated, especially in the way in which these regions interact [19]. Posterior cingulate cortex (PCC), precuneus, 


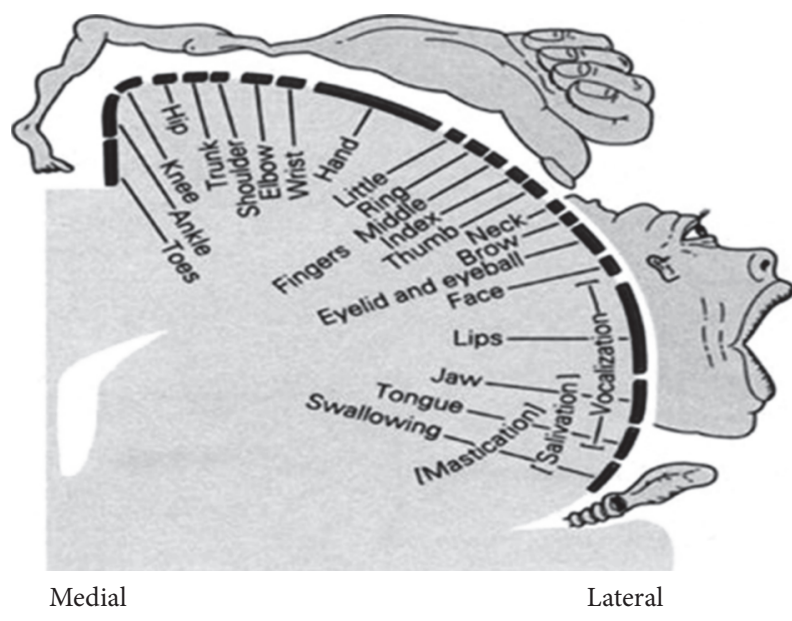

FIgUre 4: Penfield and Rasmussen's homunculus.

and parahippocampal gyrus are known to be related to the brain's default mode network (DMN), which is defined as a set of regions that is spontaneously active during passive moments [20] and associated with affective processing, memory, and self-projective thinking [21]. There was also a study reporting that Bell's palsy would bring patients negative emotion [22]. It might be the reason for activated DMN. Culmen is one part of the cerebellum which plays an important role in the motor control. It may also be involved in some cognitive function such as attention and language and in regulating fear and pleasure responses [14, 23]. There was a study reported that cerebellum was activated during functional recovery from transient peripheral motor paralysis, which was in accordance with our results [18].

Another remarkable characteristic of the results is that the decreased activation of $\mathrm{fMRI}$ as conducting lip pursing movements mainly located on the ipsilateral to the paretic side; it may be due to compensatory mechanism of brain function. However, the increased activation with finger movements located on the bilateral cerebral hemispheres. Contralateral SI and MI were activated with finger movements. A task-state fMRI study during facial and mouth movements also found activated areas were contralateral to the facial palsy even after clinical recovery [15]. It was explained by the brain's reaction to the failure of facial muscle movement. It can suggest that facial function has not completely recovered whereas the clinical assessment did not show an impairment of facial movements.

\subsection{Relationship of Hand Motor Area and Facial Motor} Area. It was also detected there were increased activation during finger movements and decreased activation during lip pursing movements, which demonstrated that cerebral blood flow in facial motor area of patients recovered from BP was reduced, while cerebral blood flow in hand motor area of patients was enhanced compared to healthy volunteers. Locations of hand and facial representation areas are neighbouring in the primary motor cortex (Figure 4). There were many studies reporting cortical reorganization of these two neighbouring areas. For example, Florence et al. [24] reported that, in the somatosensory thalamus and cortex of monkeys after accidental forelimb amputations, the forelimb representation in the ventroposterior nucleus became completely reactivated by intact inputs from the stump of the arm and from the face. Cohen et al. [25] also used functional magnetic resonance imaging to study brain activity to vibratory stimulation and voluntary movements of body parts above and below the lesion and found that no response to vibratory stimulation of the hand was observed in the primary somatosensory cortex (SI) hand area, which was conversely recruited during tongue movements that normally evoke responses only in the more lateral face area, which suggested the activated hand representation area had extended facial motor area. In studies of Bell's palsy, Rijntjes et al. [12] also found that patients with facial palsy activated a larger part of the cortex than normal volunteers when making fractionated finger movements, as measured with PET and TMS. It inferred that hand representation area extended into the orofacial area. It was mostly corresponding to our results. It might be the reason for distal end points which were used to acupuncture treatment. Not only have points located on the face been used, but also points, such as Hegu (L14), which is located on the hand, have been used in acupuncture treatment for Bell's palsy.

\section{Limitation of This Study}

Our results indicated that cortical reorganization existed in the early recovery stage of BP. The evidence provided in this research for the relationship of cortical reorganization with acupuncture treatment is limited. Therefore, we cannot exclude the possibility that the results might just reflect the self-recovery of Bell's palsy. This study tried to light the underlying mechanism of recovery of BP, although further researches are still needed. Although considering the probability that severity of BP in acute phase and duration might have an influence on the cortical reorganization, there was no abundant data to analyze their relationship. This valuable research will be carried out after we collected enough data.

\section{Conclusions and Perspective}

We have concluded evidence that functional status in the brain of patients recovered from Bell's palsy differed from those in healthy control group when making finger movements and lip pursing movements. The changed activation between the two groups included motor association cortex and cerebellum. All of these changes in the cortex might be relevant to the differences in the brain functional status. Therefore, we propose that cortical reorganization continued at different pathological stages in patients with Bell's palsy besides recovered stage. And further evidence was still needed to support our proposition.

\section{Competing Interests}

The authors declare that there are no competing interests regarding the publication of this paper. 


\section{Acknowledgments}

This paper was supported by the Project for the National Key Basic Research and Development Program (973) under Grant no. 2010CB530500 and Key Science and Technology National Program of Anhui Province under Grant no. 1604b0602020.

\section{References}

[1] M. Artzi, S. I. Shiran, M. Weinstein et al., "Cortical reorganization following injury early in life," Neural Plasticity, vol. 2016, Article ID 8615872, 9 pages, 2016.

[2] J. Cai, Q. Ji, R. Xin et al., "Contralesional cortical structural reorganization contributes to motor recovery after sub-cortical stroke: a longitudinal voxel-based morphometry study," Frontiers in Human Neuroscience, vol. 10, article 393, 2016.

[3] M. Stropahl, L. C. Chen, and S. Debener, "Cortical reorganization in postlingually deaf cochlear implant users: intra-modal and cross-modal considerations," Hearing Research, 2016.

[4] K. S. Taylor, D. J. Anastakis, and K. D. Davis, "Cutting your nerve changes your brain," Brain, vol. 132, no. 11, pp. 3122-3133, 2009.

[5] K. Vakharia and K. Vakharia, "Bell's palsy," Facial Plastic Surgery Clinics of North America, vol. 24, no. 1, pp. 1-10, 2016.

[6] J. I. Kim, M. S. Lee, T.-Y. Choi, H. Lee, and H.-J. Kwon, "Acupuncture for Bell's palsy: a systematic review and metaanalysis," Chinese Journal of Integrative Medicine, vol. 18, no. 1, pp. 48-55, 2012.

[7] E. Peitersen, "Bell's palsy: the spontaneous course of 2,500 peripheral facial nerve palsies of different etiologies," Acta OtoLaryngologica, Supplement, no. 549, pp. 4-30, 2002.

[8] R. Garmi, D. Labbé, O. Coskun, J.-F. Compère, and H. Bénateau, "Lengthening temporalis myoplasty and brain plasticity: A Functional Magnetic Resonance Imaging Study," Annales de Chirurgie Plastique et Esthetique, vol. 58, no. 4, pp. 271-276, 2013.

[9] T. Bitter, B. Sorger, V. Hesselmann, B. Krug, K. Lackner, and O. Guntinas-Lichius, "Cortical representation sites of mimic movements after facial nerve reconstruction: a functional magnetic resonance imaging study," The Laryngoscope, vol. 121, no. 4, pp. 699-706, 2011.

[10] H. Wu, H. Kan, C. Li et al., "Effect of acupuncture on functional connectivity of anterior cingulate cortex for bell's palsy patients with different clinical duration," Evidence-based Complementary and Alternative Medicine, vol. 2015, Article ID 646872, 7 pages, 2015.

[11] F. N. Yalcindag and C. Alay, "Bell's palsy during interferon alpha 2a treatment in a case with Behcet uveitis," F1000Research, vol. 2, article 245, 2013.

[12] M. Rijntjes, M. Tegenthoff, J. Liepert et al., "Cortical reorganization in patients with facial palsy," Annals of Neurology, vol. 41, no. 5, pp. 621-630, 1997.

[13] M. Wong and Y. Ming, "Correspondence on "effect of acupuncture on the brain in children with spastic cerebral palsy using functional neuroimaging (fMRI)",' Journal of Child Neurology, vol. 24, no. 10, pp. 1324-1325, 2009.

[14] S. Hu, Y. Wu, C. Li et al., "Increasing functional connectivity of the anterior cingulate cortex during the course of recovery from Bell's palsy," Neuroreport, vol. 26, no. 1, pp. 6-12, 2015.

[15] C. M. Klingner, G. F. Volk, A. Maertin et al., "Cortical reorganization in Bell's palsy," Restorative Neurology and Neuroscience, vol. 29, no. 3, pp. 203-214, 2011.
[16] X. He, Y. Zhu, C. Li et al., "Acupuncture-induced changes in functional connectivity of the primary somatosensory cortex varied with pathological stages of Bell's palsy," NeuroReport, vol. 25, no. 14, pp. 1162-1168, 2014.

[17] V. Hesselmann, R. Girnus, C. Wedekind et al., "Functional MRI using multiple receiver coils: BOLD signal changes and signalto-noise ratio for three-dimensional-PRESTO vs. single shot EPI in comparison to a standard quadrature head coil," Journal of Magnetic Resonance Imaging, vol. 20, no. 2, pp. 321-326, 2004.

[18] A. Smit, J. van der Geest, M. Metselaar, A. van der Lugt, F. VanderWerf, and C. De Zeeuw, "Long-term changes in cerebellar activation during functional recovery from transient peripheral motor paralysis," Experimental Neurology, vol. 226, no. 1, pp. 33-39, 2010.

[19] M. P. van den Heuvel and H. E. Hulshoff Pol, "Exploring the brain network: a review on resting-state fMRI functional connectivity," European Neuropsychopharmacology, vol. 20, no. 8, pp. 519-534, 2010.

[20] R. L. Buckner, "The brain's default network: origins and implications for the study of psychosis," Dialogues in Clinical Neuroscience, vol. 15, no. 3, pp. 351-358, 2013.

[21] A. Otti and M. Noll-Hussong, "Acupuncture-induced pain relief and the human brain's default mode network-an extended view of central effects of acupuncture analgesia," Forschende Komplementarmedizin, vol. 19, no. 4, pp. 197-201, 2012.

[22] L. Fu, C. Bundy, and S. A. Sadiq, "Psychological distress in people with disfigurement from facial palsy," Eye, vol. 25, no. 10, pp. 1322-1326, 2011.

[23] C. J. Stoodley, E. M. Valera, and J. D. Schmahmann, "Functional topography of the cerebellum for motor and cognitive tasks: an fMRI study," NeuroImage, vol. 59, no. 2, pp. 1560-1570, 2012.

[24] S. L. Florence, T. A. Hackett, and F. Strata, "Thalamic and cortical contributions to neural plasticity after limb amputation," Journal of Neurophysiology, vol. 83, no. 5, pp. 3154-3159, 2000.

[25] L. G. Cohen, P. Celnik, A. Pascual-Leone et al., "Functional relevance of cross-modal plasticity in blind humans," Nature, vol. 389, no. 6647, pp. 180-183, 1997. 

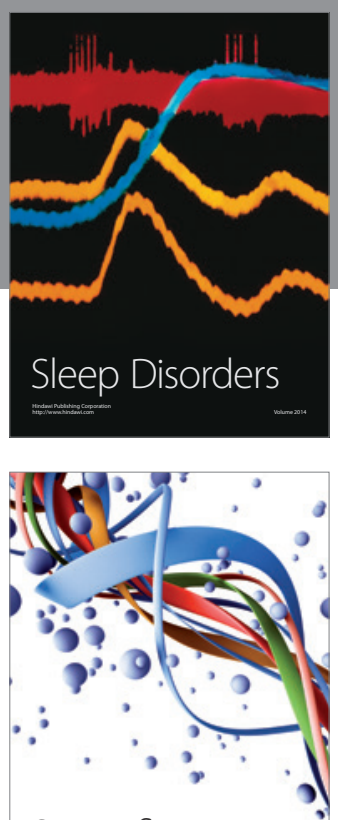

Scientifica
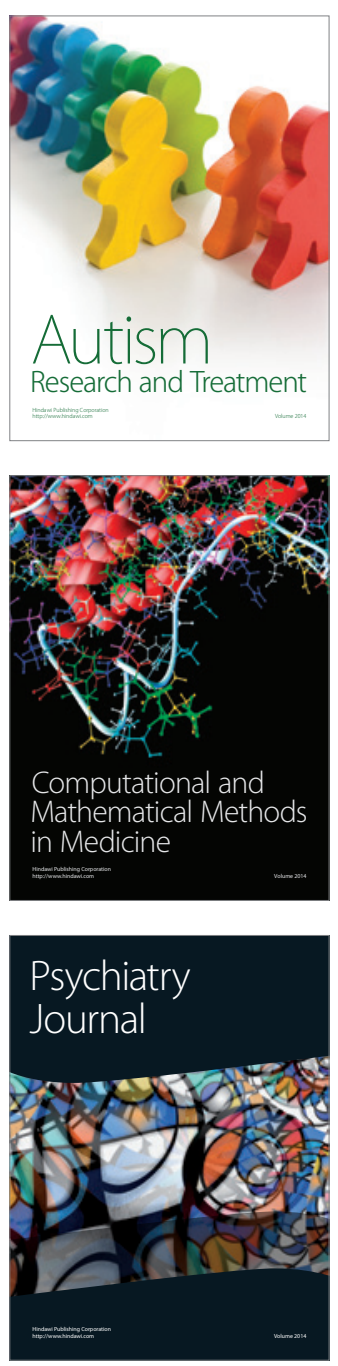
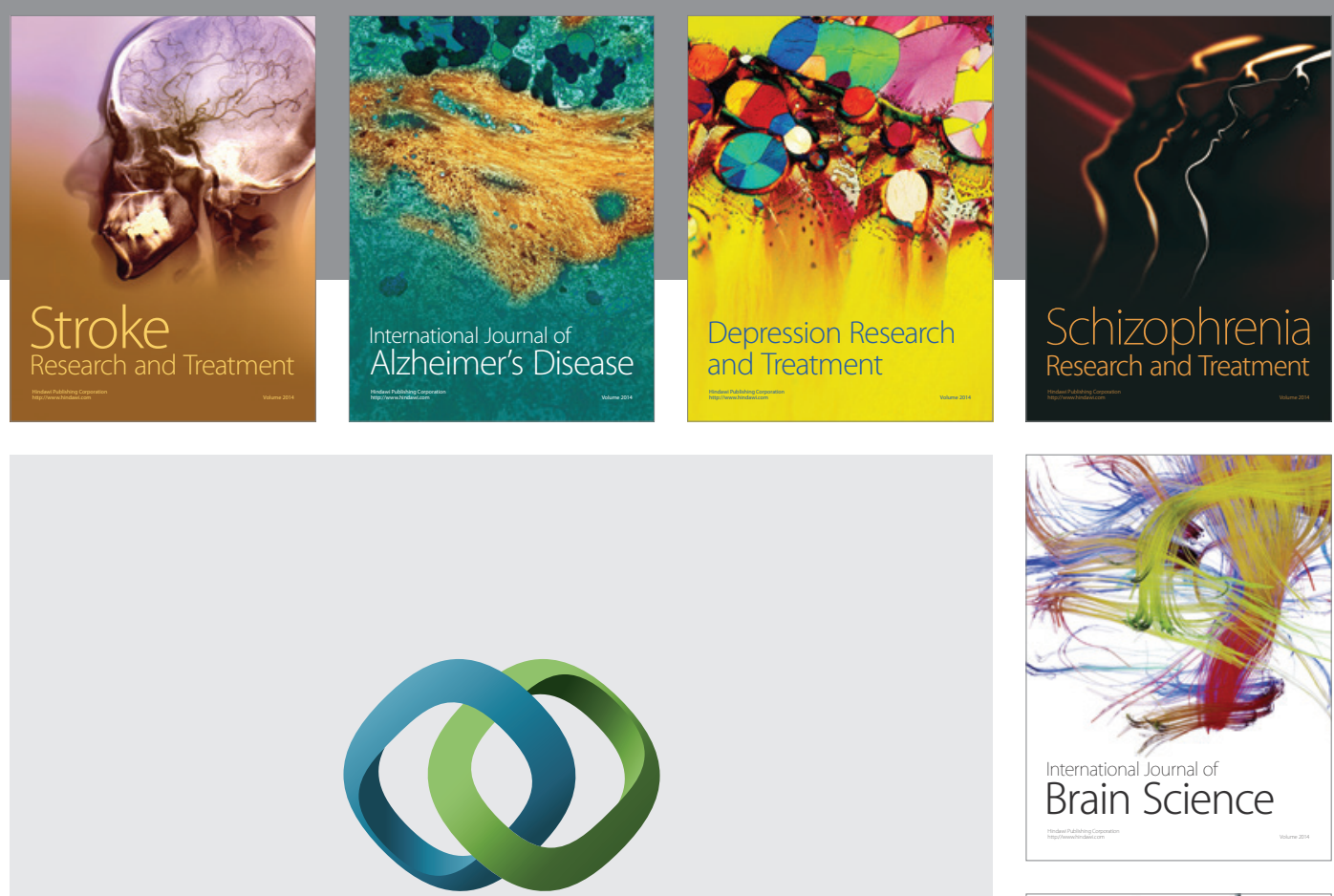

\section{Hindawi}

Submit your manuscripts at

http://www.hindawi.com
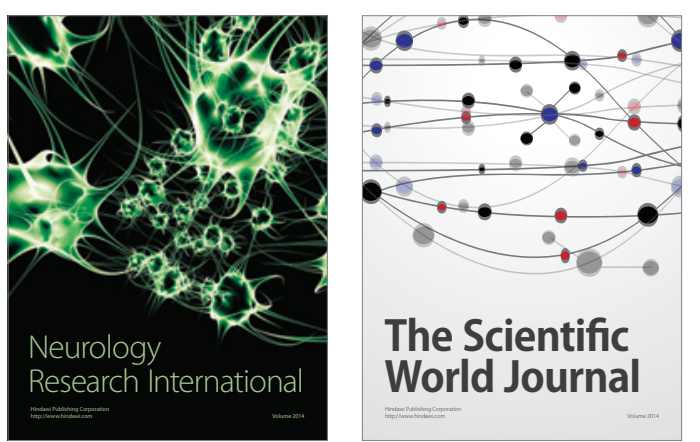

The Scientific World Journal

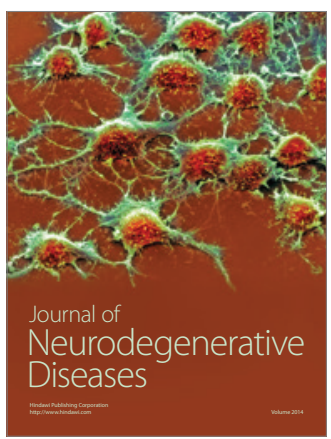

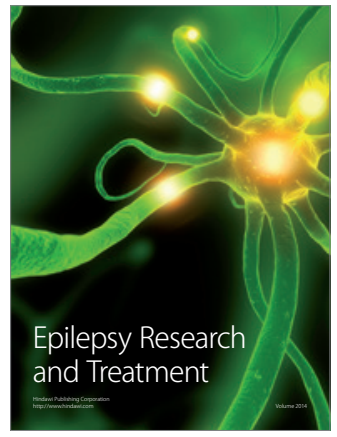

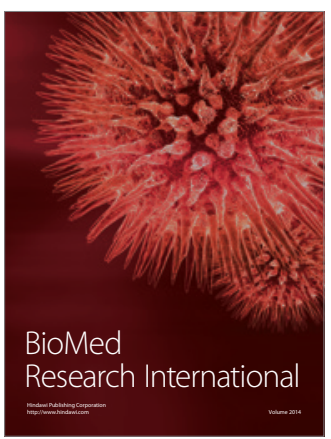

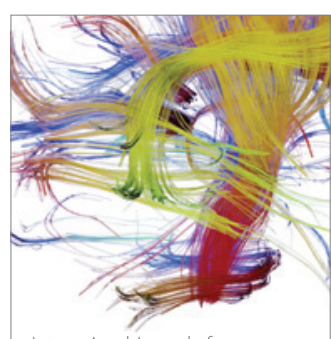

Brain Science

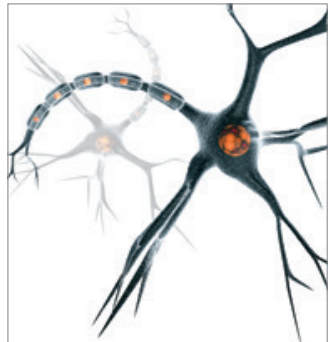

Neural Plasticity
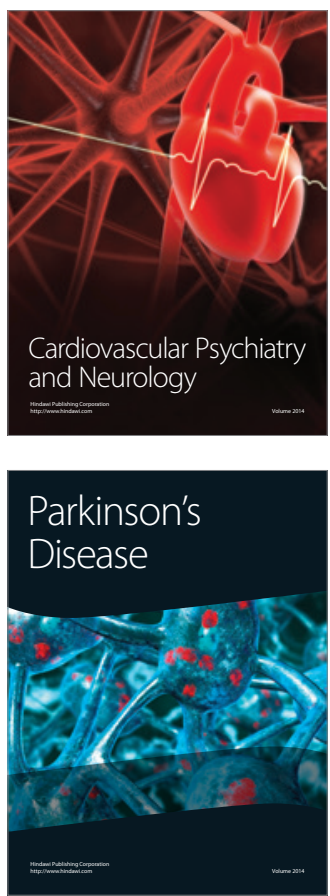